\title{
ChemComm
}

\section{Multilayered nanocoatings incorporating superparamagnetic nanoparticles for tracking of pancreatic islet transplants with magnetic resonance imaging $\dagger$}

Cite this: Chem. Commun., 2013, 49, 7255

Received 10th May 2013,

Accepted 19th June 2013

DOI: $10.1039 /$ c3cc43512a

www.rsc.org/chemcomm

\author{
Yang Wang, ${ }^{a}$ Cristina Blanco-Andujar, ${ }^{b}$ Zheng-liang Zhi, ${ }^{* a}$ Po-Wah So, \\ Nguyen Thi Kim Thanh ${ }^{b}$ and John C. Pickup ${ }^{a}$
}

A novel strategy for delivering functionalised superparamagnetic iron oxide nanoparticles to the outer surface of pancreatic islet grafts, using chemically modified polymeric nanolayers, has been developed for tracking of engrafted pancreatic islets by magnetic resonance imaging.

Research towards cell therapies has shown a growing interest in the development of noninvasive monitoring strategies using magnetic resonance imaging (MRI) as a means of assessing the functional dynamics of cell-based implants. ${ }^{1}$ In pancreatic islet transplantation, for example, current monitoring of implanted islet function mainly focuses on measuring blood glucose and insulin/C-peptide concentrations and HbA1c levels in the host. ${ }^{2}$ These measurements only provide information on the later stages of graft rejection; they cannot predict if the graft will continue to function at its current level nor can they predict for how long or indeed the continued presence of the grafted islets themselves following implantation. MRI appears to be the most promising and reliable modality for post-transplant noninvasive monitoring of the islet graft ${ }^{3}$ and its rejection. ${ }^{4}$

The key challenge in the establishment of MRI monitoring is the development of efficient chemistries for pre-labelling islet cells using magnetic nanoparticle probes which will remain stable post-transplantion and in the in vivo environment. ${ }^{5}$ Previous attempts at labelling islets using magnetic nanoparticles as the contrast agent have focused on cellular up-take of iron oxides through pre-incubating islets with a nanoparticle suspension in the culture medium for long periods (up to $24-48 \mathrm{~h}$ ). ${ }^{6}$ While these methods are elegant and often effective, the intracellular

\footnotetext{
${ }^{a}$ Diabetes Research Group, King's College London School of Medicine, Guy's Hospital Campus, London SE1 1UL, UK. E-mail: z.zhi@kcl.ac.uk

${ }^{b}$ Department of Physics and Astronomy, University College London, London, UK 'Preclinical Imaging Unit, Department of Neuroimaging, Institute of Psychiatry, King's College London, UK

† Electronic supplementary information (ESI) available: Experimental details, nanoparticle size distribution, effects of coatings on islets, monitoring of blood glucose levels, islet transplantation and histological analysis of the islet bearing tissues. See DOI: 10.1039/c3cc43512a
}

iron uptake occurs via random endocytosis, and is unpredictable and potentially toxic to cells, impairing cell viability and insulin-producing function, as well as MRI-imaging ability. ${ }^{6}$ On the other hand, surface-labelling of islets with magnetic nanoparticles by covalent-linkage has been shown recently to provide better imaging sensitivity. ${ }^{7}$ Separately, we have also showed the surface-labelling of biotinylated tumour and stem cells with magnetic nanoparticles for MRI tracking. ${ }^{8}$

In an effort to find more efficient and controllable ways of labelling islet cells with nanoparticles, we sought to develop a novel strategy to attach superparamagnetic iron oxide (SPIO) nanoparticles to the surface of islet grafts using chemically modified polymer nanolayers for non-invasive tracking of the engrafted islets by MRI. Our hypothesis was that multilayered nano-coatings incorporating SPIO nanoparticles can be attached onto islet surfaces and remain stable when they are transplanted in animals, and the nanoparticle probes will then permit MRI of the islet transplants. We believe that this approach, in combination with our anti-rejection nanocoating strategies for islet transplantation, ${ }^{9}$ will facilitate the transition of imaging of cellular transplants to clinical applications.

We generated conformal nano-thin films encapsulating islets individually using the method of layer-by-layer polyelectrolyte nano-assembly, ${ }^{9}$ the biggest advantage of which is that it allows different building materials, including polyelectrolytes and charged nanoparticles, to be entrapped between the layers to construct the composite nanofilms. ${ }^{10}$ We began our studies with the design of a suitable layer-by-layer deposition scheme for incorporating SPIO nanoparticles in nanolayers encapsulating islets that could be implanted in vivo in the same way as the normal islets.

The foundation of layer-by-layer deposition is electrostatic attraction and complex formation between oppositely charged species, which allows successive 'layers' to be built up on a solid substrate. In this study, positively charged, chemically modified chitosan and negatively charged heparin or SPIO nanoparticles can associate sequentially with the negatively charged cell surfaces in cell suspension (Fig. 1). We used chemically 


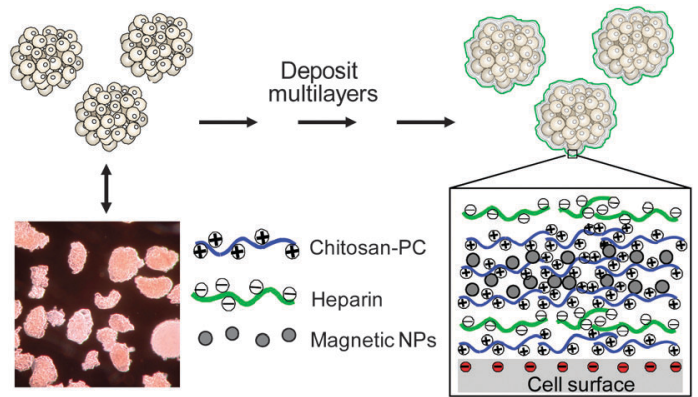

Fig. 1 Scheme of nano-encapsulation of islets in nanoengineered polysaccharide capsules. SPIO nanoparticles were incorporated into the coating construct during the successive multilayer build-up. NPs: nanoparticle probes.

modified chitosan ( $\sim 40 \%$ of the amino groups linked with phosphorylcholine [PC]) to improve solubility of the polysaccharide under physiological $\mathrm{pH}$ conditions, and to confer biocompatibility to the coatings. ${ }^{11}$ The PC moiety confers hydrophilicity, resistance to non-specific protein adsorption, and anti-coagulatory properties, all of which should enhance islet survival after transplantation. ${ }^{12}$ The charged units on chitosan, heparin and SPIO nanoparticles provide a large number of binding sites for the building materials, ensuring strong affinity to the negative charged cell surfaces. The charge reversal on the film after addition of differently charged layers makes the multilayer formation possible. The formed nanofilms were strengthened by further deposition of a chitosan/ heparin-poly-aldehyde bilayer on the top of the coating which forms cross-links with the amino group of the polysaccharides through the aldehyde group (for the reaction scheme see ESI, $\dagger$ Scheme S1).

SPIO nanoparticles which were stabilised with a citric acid coating were prepared by a modified procedure published previously. ${ }^{13}$ The SPIO nanoparticles were dark-brown coloured and exhibited magnetic response when exposed to a permanent magnet. The morphology, particle size and particle distribution

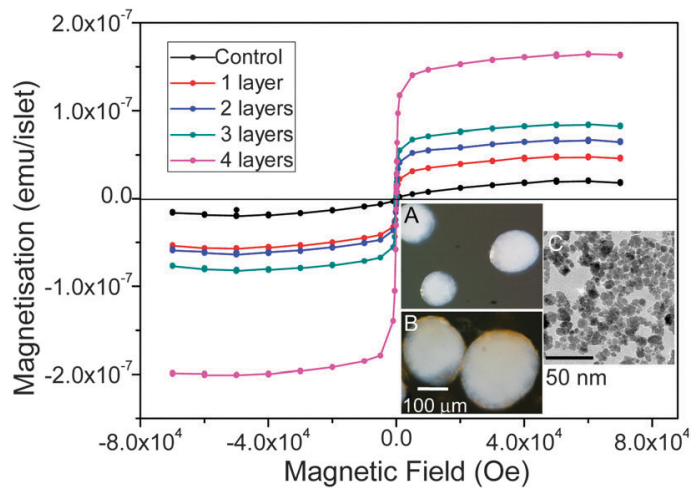

Fig. 2 SQUID measurement of iron content of islets coated with different numbers of the nanoparticle layers. Iron oxide loading: 1 layer, 543; 2 layers, 666; 3 layers, 863, and 4 layers 1967 pg per islet. Control: islets without coating. Insets: $(A)$ reflected light microscopic images of the control and (B) the nanocoated islets with 2 layers of the SPIO nanoparticles incorporated; dark brown indicates the deposited iron oxide on the islet surface. It is worthy of note that due to the 3-D nature of the islets, nanoparticles located in the centre of an islet cannot be imaged properly; (C) TEM image of the SPIO nanoparticles. were determined by transmission electron microscopy (TEM). The particles had a spheroidal shape (Fig. 2, inset C) with a mean size of $12.8 \pm 2.6 \mathrm{~nm}$ (ESI, $\dagger$ Fig. S1). The surface charge was $-35.6 \mathrm{mV}$ as examined by the $\xi$-potential measurement.

Before the SPIO nanoparticles were assembled on the islet surfaces, islets were first coated with two layers of chitosan-PC and heparin as the seed layers. Initially, we studied whether the seed coatings altered the structure of the islets by investigating islet morphological changes after coating with two combinations of the coating materials, chitosan-PC/heparin and poly-L-lysine/heparin, over a period of 7 days in a suspension culture. The poly-L-lysine/heparin coating resulted in apparent morphological damage to the islets after 3 or 7 days of culture, with most $(60-80 \%)$ of the islets disintegrating during the culture (ESI, $\uparrow$ Fig. S2). Further, FITC-fluorescently labelled poly-L-lysine appeared to accumulate around some regions, indicating unstable coverage. In contrast, islets coated with the FITC-labelled chitosan-PC/heparin layers were found to be mostly intact, indicating that the coating did not affect the islets morphologically throughout the 7 days of culture.

We then assessed if the nanoparticles could be attached to the surfaces of islets with an iron oxide loading that can be precisely controlled by the layer-by-layer scheme. To do this, we deposited a variable number of SPIO nanoparticle layer coating on the islets on top of the two seed layers. A superconducting quantum interference device-vibrating sample magnetometer (SQUID-VSM) was used to determine the quantity of attached iron oxide after each repeating bilayer (nanoparticle/chitosanPC) deposition. As shown in Fig. 2, the iron oxide loading on islets increased with increased number of bilayers, from $543 \mathrm{pg}$ $\mathrm{Fe}_{3} \mathrm{O}_{4}$ per islet for 1 bilayer, to 1967 pg $\mathrm{Fe}_{3} \mathrm{O}_{4}$ per islet for 4 bilayers. The last one gained more mass due to the exponential increase of the thickness during the film deposition. ${ }^{14}$ Moreover, the presence of SPIO nanoparticles on the islets can be observed via optical imaging due to the inherent brown coloration of the islets after the coating process. The coated islets presented a brown stain on the surface after a 2-layer nanoparticle deposition (Fig. 2, insets A and B), as observed by refractive microscopy, which confirmed the presence of the nanoparticles on the islets.

After coating of islets with nanolayers incorporating SPIO nanoparticles, we next sought to prove that the deposition of the nanoparticles on the islet surfaces did not alter their insulin-producing function in vitro. Dynamic insulin secretion of the nanocoated islets was studied and compared with the control using a perifusion system that simulates physiological conditions (ESI, $\uparrow$ Fig. S3). The six-layer (including 1 nanoparticle layer) coated mouse islets retained glucose-induced insulin secretory responses. Increasing the glucose concentration from 2 to $20 \mathrm{mmol} \mathrm{L}^{-1}$ induced a rapid, biphasic and reversible stimulation of insulin secretion from the islets, with identical or similar response patterns to the control islets, although a delay of $2 \mathrm{~min}$ in the kinetics was observed which might be due to the layers impairing the release of insulin. Increasing the nanofilm thickness to eight layers (including 2 SPIO nanoparticle layers) caused a further small reduction in the kinetics and overall amount of insulin released, but the 

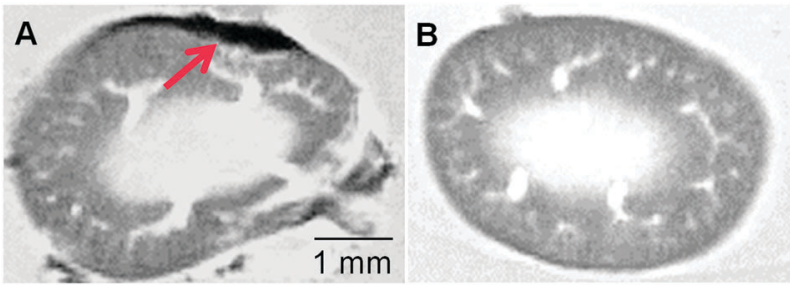

Fig. 3 Ex vivo MR images of the (A) SPIO (with two layers)-labelled islet graftbearing kidney and (B) the kidney with unlabelled islets (control). The hypointense spot as indicated by the red arrow represents the SPIO labeled islet graft 7 days after transplantation.

pattern and reversibility of the glucose-induced response were maintained.

Potential in vitro cytotoxicity exerted by the nanoparticles and other coating materials was assessed by keeping the islets in culture for up to 5 days. The survival of the coated islets within 8 layers (including 2 layers of the nanoparticles) was assessed using a cell viability assay. The viability of the nanoparticle-loaded islet cells was equivalent to the control after 5 days of culture (ESI, $\uparrow$ Fig. S4A). This indicated that the islets tolerated nanoparticle attachment and the multi-step nanocoating process did not alter the cell viability.

In addition, no elevated apoptosis in the nanoparticlecoated islets was detected after culturing for $48 \mathrm{~h}$ at $37{ }^{\circ} \mathrm{C}$, compared with the non-coated islets, as shown in ESI,† Fig. S4b. Based on the above findings, for the in vivo experiments we adopted a layering scheme including chitosan-PC/heparin as the seed layers, with the next 4 layers being repeating chitosan-PC/ nanoparticles, and the last two layers being chitosan-PC/heparinpoly-aldehyde completing the coating.

The feasibility of imaging of the transplanted islets in diabetic mice was assessed using a syngeneic transplantation model. ${ }^{9}$ Islets isolated from C57BL/6 mice were coated with 8 layers using the layering scheme described above. Streptozotocin-induced diabetic C57BL/6 mice were implanted with 300 of the same strain mouse islets (coated, including two SPIO nanoparticle layers and non-coated controls) under the left kidney capsule, according to the procedure previously reported. ${ }^{9}$

Fig. S5 (ESI $\dagger$ ) shows the monitoring of blood glucose concentrations of the animals transplanted with the coated islets and non-coated islets. As expected, implantation of the noncoated islets as a control reversed hyperglycaemia and restored normoglycaemia after 3 days during which revasculisation occurred. Nanocoated islets with incorporated SPIO nanoparticles were equally effective in reversing hyperglycaemia, with a time course similar to that of the control islet grafts, showing good agreement with the functionality displayed in the in vitro analyses.

Due to regulatory restrictions on transport of experimental live animals, we did not perform whole-animal MRI scanning, since the scanner is at a separate designated establishment. We thus retrieved the graft-bearing kidneys by nephrectomy 7 days after transplantation. The paraformaldehyde-fixed kidney with transplanted syngeneic islets was then imaged using MRI. We found that the transplanted islets with coated SPIO nanoparticles appeared as dark hypointense zones in $\mathrm{T}_{2}$-weighted images, representing islet clusters localised at the transplant site in the left kidney (ESI, $\uparrow$ Fig. S6), as compared to the control kidney with uncoated islets (Fig. 3). This observation indicated that the transplanted islets remained associated with the kidney tissue, and that they retained the attached SPIO nanoparticles. This experiment served as proof-of-concept for the ex vivo MRI detection of surface-labelled islets after transplantation. We are currently working towards live animal scanning by MRI after transplantation in compliance with the relevant regulations and institutional guidelines.

Following MRI, we carried out subsequent histological analysis of the graft materials retrieved 7 days after implantation. The tissue sections showed the presence of an insulin-positive islet mass in the grafts, with normal patterns of immunostaining for insulin, and no apparent infiltration of leukocytes at the graft sites (ESI, $\uparrow$ Fig. S7, A and B).

In summary, we investigated the use of polyionic multilayers incorporating SPIO nanoparticles to modify islet surfaces via the layer-by-layer technique and optimised the coating composition and iron loading for minimal loss of islet function and viability. We showed that it is feasible to image, using MRI, the islet grafts under the mouse kidney capsule using the surface labelling strategy. We consider these feasibility results as promising steps towards future imaging of cellular transplants in clinical applications. More extensive examination of the in vivo stability and safety of the nanoparticle probes is, however, now needed before progressing to clinical development.

This work was supported by the Engineering and Physical Sciences Research Council (EPSRC) (UK) in a Science and Innovation award (EP/D062861/1) (J. C. Pickup). NTKT thanks the Royal Society for her University Research Fellowship.

\section{Notes and references}

1 S. Laurent, S. Boutry, I. Mahieu, L. Vander Elst and R. N. Muller, Curr. Med. Chem., 2009, 16, 4712.

2 A. M. Shapiro, J. R. Lakey, E. A. Ryan, G. S. Korbutt, E. Toth, G. L. Warnock, N. M. Kneteman and R. V. Rajotte, N. Engl. J. Med., 2000, 343, 230.

3 N. V. Evgenov, Z. Medarova, G. Dai, S. Bonner-Weir and A. Moore, Nat. Med., 2006, $12,144$.

4 N. V. Evgenov, Z. Medarova, J. Pratt, P. Pantazopoulos, S. Leyting, S. Bonner-Weir and A. Moore, Diabetes, 2006, 55, 2419.

5 K. Oishi, Y. Miyamoto, H. Saito, K. Murase, K. Ono, M. Sawada, M. Watanabe, Y. Noguchi, T. Fujiwara, S. Hayashi and H. Noguchi, PLoS One, 2013, 8, e57046.

6 F. Ris, M. Lepetit-Coiffe, P. Meda, L. A. Crowe, C. Toso, M. Armanet, N. Niclauss, G. Parnaud, L. Giovannoni, D. Bosco, P. Morel, J. P. Vallee and T. Berney, Cell Transplant., 2010, 19, 1573.

7 M. J. Jung, S. S. Lee, Y. H. Hwang, H. S. Jung, J. W. Hwang, M. J. Kim, S. Yoon and D. Y. Lee, Biomaterials, 2011, 32, 9391.

8 P. W. So, T. Kalber, D. Hunt, M. Farqharson, A. El-Ebraheem, H. G. Parkes, R. Simon and J. D. Bell, Cell Transplant., 2010, 19, 419.

9 Z. L. Zhi, A. Kerby, A. J. King, P. M. Jones and J. C. Pickup, Diabetologia, 2012, 55, 1081.

10 W. Yuan, Z. Lua and C. M. Li, J. Mater. Chem., 2011, 21, 5148.

11 Z. L. Zhi, B. Liu, P. M. Jones and J. C. Pickup, Biomacromolecules, $2010,8,610$.

12 S. Monge, B. Canniccioni, A. Graillot and J. J. Robin, Biomacromoleules, 2011, 12, 1973.

13 C. Blanco-Andujar, D. Ortega, Q. A. Pankhurst and N. T. K. Thanh, J. Mater. Chem., 2012, 22, 12498; C. Blanco-Andujar, unpublished data.

14 L. Richert, P. Lavalle, E. Payan, X. Z. Shu, G. D. Prestwich, J. F. Stoltz, P. Schaaf, J. C. Voegel and C. Picart, Langmuir, 2004, 20, 448. 